\title{
Distributional vectors encode referential attributes
}

\author{
Abhijeet Gupta* Gemma Boleda ${ }^{\dagger}$ Marco Baroni $^{\dagger}$ Sebastian Padó* \\ ${ }^{*}$ Institut für Maschinelle Sprachverarbeitung \\ Universität Stuttgart \\ ${ }^{\dagger}$ Center for Mind/Brain Sciences \\ University of Trento
}

\begin{abstract}
Distributional methods have proven to excel at capturing fuzzy, graded aspects of meaning (Italy is more similar to Spain than to Germany). In contrast, it is difficult to extract the values of more specific attributes of word referents from distributional representations, attributes of the kind typically found in structured knowledge bases (Italy has 60 million inhabitants). In this paper, we pursue the hypothesis that distributional vectors also implicitly encode referential attributes.

We show that a standard supervised regression model is in fact sufficient to retrieve such attributes to a reasonable degree of accuracy: When evaluated on the prediction of both categorical and numeric attributes of countries and cities, the model consistently reduces baseline error by $30 \%$, and is not far from the upper bound. Further analysis suggests that our model is able to "objectify" distributional representations for entities, anchoring them more firmly in the external world in measurable ways.
\end{abstract}

\section{Introduction}

Distributional models induce vector-based semantic representations of words from their contextual distributions in corpora, exploiting the observation that words with related meanings tend to occur in similar linguistic contexts (Turney and Pantel, 2010; Erk, 2012). Since the approach only requires raw text as input, it can be used to harvest word representations on a very large scale. By encoding the rich knowledge that is present in text, these representations are able to capture many aspects of word meaning. Moreover, approximating semantic similarity by graded geometric distance in a vector space is an effective strategy to address the many linguistic phenomena that are better characterized in gradient rather than discrete terms, such as synonymy, selectional preferences, and semantic priming (Baroni and Lenci, 2010; Erk et al., 2010; Padó and Lapata, 2007, among others).

However, not all aspects of human semantic knowledge are satisfactorily captured in terms of fuzzy relations and graded similarity. In particular, our knowledge of the meaning of words denoting specific entities involves a number of "hard facts" about the referents they denote that are best formalized as attribute-value pairs, of the sort that are stored in manually-curated knowledge bases, such as FreeBase or Wikidata. ${ }^{1}$ While distributional vectors can capture the useful fact that, say, Italy is in many ways more similar to Spain than to Germany, as humans we also know (or we can easily look up) a set of objective facts about Italy, such as what is its capital, its area, its official language and GDP, that are difficult to express in the language of vector algebra and geometry.

In this paper, we explore the hypothesis that distributional vectors implicitly encode such attributes of referential entities, which we will call referential attributes. We show that a simple supervised algorithm applied to vectors can retrieve them so that they can be expressed in the explicit language of structured knowledge bases. Concretely, we train a logistic regression model to predict the values of both numeric and categorical FreeBase attributes of countries and cities from their distributional vectors. This model makes predictions that are significantly better than an informed baseline, in-between the latter and an upper-bound method. Qualitative analysis of the results points both to the inherent difficulty of correctly retrieving certain classes of attributes, and to some intriguing properties of the conceptual nature of the knowledge encoded in distributional data, that bias their predictions about certain objective attributes of geographic entities.

\footnotetext{
${ }^{1}$ www. freebase.com, www.wikidata.org.
} 
We see our experiment as a first step towards integrating conceptual and referential aspects of meaning in distributional semantics, as we further discuss in the conclusion.

\section{Method}

\subsection{Distributional Representations}

Mikolov et al.'s (2013) skip-gram model is a state-of-the-art "predictive" distributional semantic model which represents each word in a space of latent dimensions optimized to predict the contexts of the word's occurrences. For our study, we adopt the pre-trained 1,000-dimensional skipgram model for Named Entities that is available at https://code.google.com/p/word2vec/ and was produced from a 100-billion token news corpus. We refer to this model as WORD2VEC.

\subsection{Referential Representations}

As our source of referential attributes, we use FreeBase (see footnote 1), a knowledge base of structured information on a wide range of entities of different semantic types (people, geographical entities, etc.). The information in FreeBase comes from various sources, including Wikipedia and domainspecific databases, plus user content generation and correction. FreeBase currently records at least 2 attributes for over 47 million entities, and it has been used fairly extensively in NLP before (Mintz et al., 2009; Socher et al., 2013a, among others).

For each entity, FreeBase contains a list of attribute-value tuples (where values can in turn be entities, allowing a graph view of the data that we do not exploit here). Table 1 shows a sample of the attributes that FreeBase records for countries. Note that some attributes are simple (e.g., date_founded), while other can be called complex, in the sense that they are attributes of attributes (e.g., geolocation: : latitude). We use a double-colon notation to refer to complex attributes. The values of all attributes can be either numeric or categorical. The numeric attributes in particular are often strongly correlated, both within attributes types across years (e.g., fertility rate in different years) and across attributes within years (e.g., absolute GDP and GDP per capita in a given year).

We built two datasets for our experiments, one for countries and one for cities, with data automatically extracted from FreeBase. ${ }^{2}$ We consider two

\footnotetext{
${ }^{2}$ Both datasets are publicly available at http:
}

\begin{tabular}{l|r} 
Attribute & Value \\
\hline geolocation: :latitude & 52.52 \\
geolocation: :longitude & 13.38 \\
fertility_rate: $: 1960$ & 2.37 \\
fertility_rate: $: 1994$ & 1.24 \\
fertility_rate: $: 2010$ & 1.39 \\
date_founded & $1871-01-18$ \\
containedBy & Western Europe \\
containedBy & Europe \\
containedBy & Eurasia \\
adjectival_form & German
\end{tabular}

Table 1: Sample of numeric and binary FreeBase attributes for Germany.

datasets in order to check that the mapping we seek can be established not just for one, possible handpicked, type of entities; we leave it to future work to study very different kinds of entities, such as people or institutions.

The Countries dataset consists of the 260 countries for which we have a distributional vector. Some countries do not exist anymore, like Yugoslavia, but, since this does not impact our method, we keep them in the dataset. The dataset records all simple attributes as well as complex attributes of at most two hops in the FreeBase graph, without manual inspection. We linearly rescale all numeric attributes to [0..1] and translate all categorical attributes into a binary representation by suffixing the original value to the original attribute name. For example, the attribute member-of: :organization with the value world_bank results in a binary attribute member-of: : organization: : world_bank

having value 1 for all and only those countries that are members of the World Bank, 0 for the others. ${ }^{3}$ Attributes that occur less than 15 times are discarded, since they are either not consistently recorded or rare. This results in a total of 707 numeric and 247 binary attributes. Finally, we partition the data into training, validation, and test set, using a 60-20-20 percent split.

We apply the same process to the Cities dataset, which consists of 1645 cities from the intersection of the distributional and FreeBase city lists. In

\footnotetext{
//www.ims.uni-stuttgart.de/forschung/ ressourcen/korpora/CityCountry.html.

${ }^{3} \mathrm{We}$ considered treating some categorical attributes as multi-valued, but decided against it since the cases in which alternative values are mutually exclusive are rare (e.g., the same country can be containedBy multiple entities, cf. Table 1).
} 
this case, we have 211 numeric and 106 binary attributes - the numbers are smaller because countries have a richer representation in FreeBase than cities.

\subsection{Attribute Prediction}

We do zero-shot learning of full FreeBase attributebased country/city representations, based on distributional (WORD2VEC) representations. It is zeroshot learning in the sense of Palatucci et al. (2009): We split the datasets at the entity, rather than attribute level, such that at test time our system must predict the full attribute set of countries and cities that were not seen during training at all.

We use logistic regression. In effect, we predict each output variable (FreeBase attribute) with an independent logistic regression model based on a constant set of input features (WORD2VEC distributional dimensions). We call this model DisT2REF. DIST2REF does not take advantage of the correlations between the output attributes mentioned in Section 2.2.

The dependent variables are binary as well as numeric FreeBase attributes, and our model does not distinguish between them. For binary attributes, we interpret the value returned by the model as the probability of "success" of a binary Bernoulli trial. In the numeric case, we view the probability returned by the model as directly representing normalized attribute values.

\subsection{Experimental Setup}

We design the model using the Countries dataset, and apply it to Cities without further tuning to test its robustness. We optimize the parameters with gradient descent, using the Cross Entropy error function. We considered $\mathrm{L}_{2}$ regularization to address possible overfitting, but experiments on validation set showed that the model performs best without any regularization.

As for baselines, for binary features we predict the majority class ( 0 or 1$)$, and for numeric features we predict the mean value of the feature in the training set. These are of course strong baselines to beat.

As an upper bound, we train a model that uses the same architecture as described above but uses as input not distributional vectors but the FreeBase attributes themselves. In other words, this model has to learn "only" an identity mapping. This is not trivial, though, for example due to the presence of strong correlations among attributes, in particular the time series attributes (cf. Section 2.2). We call this model REF2REF.

\subsection{Evaluation}

Since there is no appropriate unified evaluation measure that covers both numeric and binary attributes, we evaluate them separately. For binary attributes, we report the attributes' mean accuracy.

For numeric attributes, we consider attribute prediction a ranking task. As an example, take the population::2011: :number attribute, and imagine that we only have three countries (Germany: 80M; Spain: 36M; and Netherlands: 17M). If we predict 56M for Spain's population, it is still (correctly) predicted as the second most populous country (rank difference of 0 ); a prediction of $16 \mathrm{M}$, however, would push Spain to third place (rank difference of 1).

This suggests the use of rank correlation coefficients like Spearman's $\rho$. However, we want to measure not only how well the model can rank the countries in the test set, but also whether these predictions are consistent with the training set (which makes evaluation both more challenging and more realistic). One way of achieving this goal would be to use $\rho$ on the union of training and test instances, but this could lead to misleadingly high correlation coefficients since this method would include the labels of the training instances in the evaluation.

Consequently, we define our own evaluation measure, following a rationale similar to Frome et al.'s (2013) evaluation of a zero-shot learning scenario. What we evaluate, for each attribute, is the rank of the test countries in the whole country list. Note that this makes our task harder, as there are more confounders: If we only evaluated on the test set, there would be shorter lists and therefore less chances of getting bad rankings. So, concretely, we first define the prediction quality of each attribute, $Q(a)$, as the median of the rank difference between the prediction and the gold standard in a list that includes both training and test countries (we use the median to give less weight to outlier countries). We also normalize the rank difference to obtain a number between zero and one. In a second step, we define the quality of the complete model, the normalized rank score (NRS), as the mean of all attribute quality scores, in parallel to our evaluation on binary attributes.

Let the set of instances $I$ be partitioned into training instances $T r$ and test instances $T s$. Let $a \in A$ 


\begin{tabular}{ll|cc} 
Attribute Type & Model & Countries & Cities \\
\hline \multirow{3}{*}{ Binary (Acc) } & Most Frequent Class Baseline & 0.86 & 0.97 \\
& DisT2REF & 0.90 & 0.99 \\
& REF2REF (upper bound) & 0.96 & 1.00 \\
\hline \multirow{3}{*}{ Numeric (NRS) } & Mean Value Baseline & 0.35 & 0.35 \\
& DisT2REF & 0.22 & 0.25 \\
& REF2REF (upper bound) & 0.14 & 0.21
\end{tabular}

Table 2: Results for predicting FreeBase attributes from distributional vectors on the test sets. Both evaluation measures range between 0 and 1. For accuracy, 1 is best. For normalized rank score (NRS), 0 is best. All pairwise differences between models are significant $(\mathrm{p}<0.001$, bootstrap resampling).

denote an attribute. We write $p_{a}(i)$ for the predicted value of attribute $a$ for instance $i$ and $g_{a}(i)$ for the gold standard value. Finally, let $r(v, S)$ denote the rank of value $v$ in the list resulting when ordering the set $S$. Now we can define:

$$
\begin{aligned}
Q(a) & =\frac{1}{\|I\|} \operatorname{med}\left\{\mid r\left(p_{a}(i), I\right)-\right. \\
N R S & \left.=\frac{1}{\|A\|} \sum_{a \in A} Q\left(g_{a}(i), I\right)|-1| i \in T s\right\}
\end{aligned}
$$

This measure can be interpreted similarly to Mean Reciprocal Rank (Manning et al., 2008): It has range [0..1], with smaller numbers indicating better ranking: 0.1 , for example, means that, on average, the prediction is $10 \%$ of the ranks off (e.g., by four countries in a forty-country list). ${ }^{4}$

Note that, when evaluating each instance $i$, we use gold-standard values for all other instances, so that there the baseline is not hampered by ties.

\section{Results}

Table 2 shows the results of our experiments on the two test sets. For accuracy 1 is best, but for NRS 0 is best. Recall from Section 2.2 that we perform model selection on the Countries dataset only.

The baseline is relatively high, in particular for the binary attributes, many of which are positive for a small subset of entities only. The amount of skew differs considerably between the two datasets, though. For Countries, the baseline yields an accuracy of 0.86 , but it achieves 0.97 on Cities. The increase stems from very sparse categorical City features such as containedBy, which includes all

\footnotetext{
${ }^{4}$ Subtracting 1 in Equation (1) ensures that, when the predicted and gold value of an attribute are adjacent in the ranking, their rank difference is 0 , capturing the intuition of rank difference as counting the number of falsely intervening items.
}

levels of administrative divisions - that is, for the US, all counties appear as values and are transformed into sparse binary features (cf. Section 2.2). Of course, the predictions of the baseline are useless, since it always predicts the absence of any features. On numeric features, where the baseline predicts the mean, its performance is $0.35 \mathrm{NRS}$ on both datasets. In other words, its average prediction is off by about one third the length of the ranked list for each attribute.

Recall that the upper bound model, REF2REF, uses FreeBase attributes to predict FreeBase attributes. All it has to learn is that there is one feature in the input that corresponds ideally to the output. This works almost perfectly for binary attributes, with accuracy values of 0.96 (Countries) and 1.00 (Cities). However, its performance on numeric features (with NRS at 0.14 and 0.21 , respectively) is not quite perfect. We attribute this to the presence of correlations (cf. Section 2.2).

The model whose performance we are actually interested in, DIST2REF, in which we map from distributional information to FreeBase features, performs with remarkable consistency between these two extremes. In fact, we see a consistent error reduction of around $30 \%$ over the baseline, with a similar distance to the upper bound. A significance test with bootstrap resampling (Efron and Tibshirani, 1994) showed that all pairwise comparisons (Baseline vs. DIST2ReF, DIST2ReF vs. REF2REF) are statistically significant at $\mathrm{p}<0.001$.

To rule out that we misinterpret our accuracybased evaluation for the binary features in the face of a highly skewed class distribution, we also computed precision, recall, and F-Score values. The relative patterns match those of the accuracybased evaluation well (Countries: baseline $\mathrm{F}=0.13$, DIST2REF F=0.51, REF2REF F=0.77) and indicate that generally precision is higher than recall. 
We think that these are overall promising results, given that the FreeBase attributes we predict are fairly fine-grained, and we only use generic distributional information as input.

\section{Analysis}

We take the overall results just presented to suggest that we are able to learn referential attributes from distributional information to a large extent. In this section we take a closer look at what kind of information we are able to learn, what is beyond the scope of our model, and what are the differences between the entity representations in WORD2VEC and the ones our model produces. All the data concerns the test sets only.

\subsection{Attribute Groups}

We start with a qualitative analysis of the Countries dataset. Due to the large number of attributes, we sort all individual attributes into attribute groups by their base name (i.e. the leftmost component of their name, cf. Section 2.2), which offers an accessible level of granularity for inspection. We obtain 34 numeric and 40 binary attribute groups with median sizes of 8.5 and 2 attributes per group, respectively.

Table 3 shows the attribute groups for both types sorted by quality. For each group, we report average normalized rank score (NRS) and accuracy, respectively, for both DIST2REF and the baseline.

The analysis suggests that there are two main factors that account for the results: (1) The degree to which an attribute is contextually supported, that is, to what extent its values can be identified on the basis of the contextual information that is captured in a distributional model, and (2) general properties of the data that affect Machine Learning, most notably data sparseness, possibly also feature value distributions.

Attributes that are contextually supported include for instance those related to socioeconomic development (see below for details); people talk (and so write) about countries being more or less developed, rich, having one or another kind of laws, and this is captured in the abstractions over textual context that distributional models perform. As an extreme example of an attribute that is not contextually supported, consider the numeric ISO code of a country (iso_numeric), whose values are arbitrary: They do not correspond to facts about the world that are reflected in the way people use lan- guage, and so can't be picked up by the distributional model. For this reason, DIST2REF does worse than the baseline.

Note that, in a sufficiently large corpus, we might indeed encounter statements like The numeric ISO code for Spain is 724 . However, since distributional models represent words as aggregated distributions of their contexts, and compute semantic similarity from these context distributions, the contexts that they use need to be generic enough to yield meaningful overlap between concepts (e.g., words). As a result, distributional models cannot easily represent knowledge of the form "the value for property $\mathrm{Y}$ of word/concept X is Z".

Fortunately, we find that many FreeBase attributes are contextually supported to a substantial degree, even some seemingly arbitrary ones. An example is calling codes, which we predict very well. They turn out to be correlated with geolocations: $2 \mathrm{X}$ calling codes are located in Africa, 3X calling codes in Southern and Eastern Europe and 4X calling codes in Western and Northern Europe (for comparison, ISO codes are assigned in a roughly alphabetical order).

Numeric Attributes. Our best numeric attributes belong to the geolocation group (latitude and longitude). We provide a more detailed analysis of these attributes below (Section 4.2). As mentioned above, we also excel at many attributes related to a country's economic and social development (broadly construed), such as GNI, GDP, $\mathrm{CO}_{2}$ emissions, internet usage (each per capita), or fertility rate. These attributes can be expected to be contextually grounded - e.g., Luxembourg will occur with contexts like "broadband" or "rich" more than India.

Note, however, that the information contained in the vectors is surprisingly subtle: For instance, the fertility rate is a function of both general development status (lower rates in more developed countries) and of specific social factors (higher rates in countries with more support for families, such as France and Finland compared countries with less support, such as Germany or Italy).

Around the middle of the table, we find the absolute versions of the developmental cluster above (GNI in \$, real and nominal GDP). Evidently, the absolute versions of these attributes are substantially less contextually supported than the relative versions. This is not surprising: While India and China have high absolute GDPs because they are 
Numeric Attributes (Normalized Rank Score: lower is better)

Binary Attributes (Accuracy: higher is better)

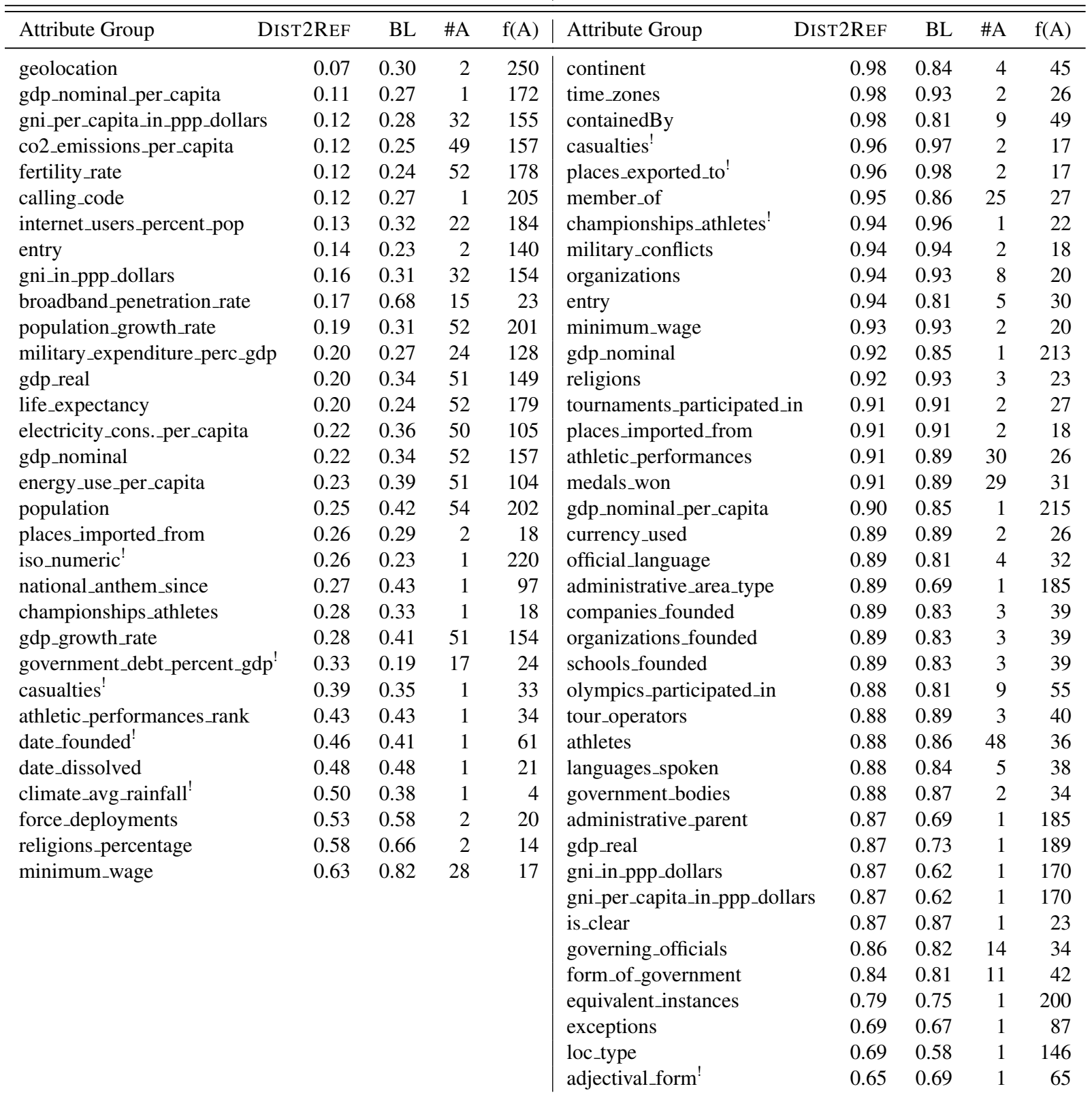

Table 3: Results for all attribute groups on the Countries test set, in descending order of performance. DisT2REF, BL: models; \#A: number of attributes in group; $\mathbf{f}(\mathbf{A})$ : median number of countries instantiating each attribute in the dataset (260 countries); !: attribute group where model performs worse than baseline.

large countries, and for instance Luxembourg has a much smaller one, these numbers are not indicative of the actual conditions in these countries, and therefore also not so clearly correlated with what people write about them. This provides another interesting angle on the difference between distributional and formal knowledge representation. In a formal system, absolute GDP, relative GDP, and population stand in a fixed linear relationship and knowing any two of the three uniquely determines the third - thus, all three attributes have equal status. In our distributional space, their status is clearly different, determined by the conceptual relevance of the different attributes.

Towards the end of the table, we find more attributes related to socioeconomic development, such as government_percent_debt and minimum_wage. While these should be contextually supported, too, the problem here is factor (2) mentioned above, namely severe data sparsity (see column $\mathbf{f}(\mathbf{A})$ in Table 3, which lists the median number of datapoints that exhibit each attribute group). The same goes for the remaining attribute groups, for instance casualties (describing the 
total number of military casualties incurred in history), date_founded and date_dissolved, ${ }^{5}$ or climate_avg_rainfall.

Binary Attributes. The binary attributes show a similar picture, albeit somewhat less sharp. We again find contextually unsupported groups, many of them arising from our fully automatic attribute mining from FreeBase (cf. Section 2.2). There are many categorical attributes that store metadata about numeric attributes (such as the currency in the gdp and gni groups) as well as meta-information of FreeBase: exceptions is a specific marker of potentially inconsistent entries about Ghana, and equivalent_instances is a flag concerning links between FreeBase and OpenCyc. Fortunately, almost all contextually unsupported groups are small, with only one or two attributes, and do not have a large impact on the overall performance. We decided not to exclude them from evaluation for robustness' sake, since there is no automatic way to identify contextually unsupported attributes in a new dataset.

We obtain good results on meaningful attributes that are arguably strongly contextually grounded, such as geographical and geopolitical attributes (member_of: membership in international organizations; location on a cont inent, etc.). However, we fare relatively badly on government-related attributes (form of government, governing officials). While this seems surprising at first glance, the form_of_government attribute in FreeBase makes very fine-grained distinctions: Its values include "unitary state", "presidential system", "parliamentary system" and "republic", which are not mutually exclusive, and misses obvious alternatives like "authoritarian system". It is not surprising that distributional models cannot make such subtle distinction between presidential and parliamentary systems. The attribute governing_official presents a similar case. Other bad attributes are very domain-specific, including athletes, encoding the athletic disciplines that countries participate in (such as swimming, judo, running, etc.), and the data sparsity issue is certainly worse for the binary attributes.

\footnotetext{
${ }^{5}$ Note that date-based attributes can be contextually supported: We do better on national_anthem_since, for which we have more datapoints, 97.
}

\begin{tabular}{l|rr} 
Model & Countries & Cities \\
\hline WorD2VEC & -0.36 & -0.45 \\
Dist2REF & 0.49 & 0.88
\end{tabular}

Table 4: Pearson correlation coefficients of modelpredicted vs. ground truth distances between countries and cities in the test sets. WORD2VEC correlations are negative because we use cosines.

\subsection{Geolocation}

To analyze the difference between the distributional representations and the output of our model, we focus on geolocation, our best attribute group.

It has already been shown that geometric distance in distributional space captures, to a certain extent, physical distance between locations in the real world (Louwerse and Zwaan, 2009). Table 4 shows that DIST2REF extracts even more precise distance information from distributional vectors. The table reports the correlation between real and model-predicted distances for countries and cities. Ground-truth great circle distances (Kern and Bland, 1948) between items are computed using the FreeBase longitude and latitude values; for DIST2REF we use its predicted latitude and longitude values; for WORD2VEC, the cosines between the corresponding distributional vectors.

We obtain highly significant correlations in all cases $\left(\mathrm{p}<10^{-14}\right)$, but much higher for DIST2REF. For countries, as shown in Table 4 , the correlation is -0.36 for WORD2VEC (negative, because cosine is a similarity measure), 0.49 for DIST2REF. For cities, WORD2VEC reaches -0.45 correlation, and DIST2REF distances are at 0.88 , showing that the method can estimate city positions to a perhaps unexpectedly high degree of accuracy. ${ }^{6}$

This result suggests that we manage to objectify the information in the distributional model, anchoring the entities more firmly in the external world. Indeed, distributional models are known to be subject to conceptual or cultural effects in their distance estimations. For instance, in WORD2VEC German and Spanish cities are much farther away than in the physical world, while cities within Spain and within Germany are predicted to be a bit closer than they actually are. Note that these effects have

\footnotetext{
${ }^{6}$ The results are confirmed when the analysis is repeated using the Spearman correlation measure: The DIST2REF coefficients are stable, whereas those of WORD2VEC go down to 0.22 (countries) and 0.40 (cities), respectively. The good results for Spearman, as a rank-based measure, indicate that our success is not dominated by outliers.
} 
an actual cognitive basis: Human intuitions about objective physical distance between countries and cities are biased by cognitive, cultural and socioeconomic factors, as explored for example in Friedman et al. (2002), who report that Texans locate Canadian cities closer to the US border relative to Mexican cities, despite their proximity to the latter, and that they place Southern US cities further south than they really are.

Interestingly, DisT2REF does also show some cultural effects in its geolocation errors: For example, some Pacific island states with lesser-known identities (e.g., Nauru and French Polynesia) are placed in the Indian Ocean, where we find the perhaps prototypes of beautiful islands, like Seychelles and Mauritius; also, Central American countries (such as Panama, El Salvador, and Nicaragua) move towards their "cultural center of gravity", South America.

However, this kind of cultural bias is much more prominent in the original WORD2VEC distributional representation. The Spain/Germany effect discussed above is not found in the DIST2REF model at all. And while both DIST2REF and WORD2VEC place Mexican and Spanish cities in our test set closer to each other than they actually are, WORD2VEC does so to a much larger extent. In line with our goal to extract referential attributes, thus, we are satisfied to see that DIST2REF manages to minimize this bias and distill the referential part from the distributional representations.

\section{Related Work}

There is a large literature on exploiting corpus evidence, sometimes through distributional semantic methods, in order to construct and populate structured knowledge bases (KBs) (e.g., Buitelaar and Cimiano (2008) and references therein). This line of work, however, does not attempt to connect entity representations extracted from corpora and from KBs, as we do. Moreover, it focuses on harvesting relations between entities or between entities and a limited number of discrete attributes, rather than predicting full-fledged KB representations of specific entities, like we do. Freitas and Curry (2014) and Freitas et al. (2014) embed relational graphs from KBs in a distributional semantic space to support various forms of search and reasoning about the KB. The focus is again on relations between discrete entities, and on exploiting distributional semantics to navigate among them.
Socher et al. (2013a) represent WordNet and FreeBase entities with corpus-based distributional vectors. They train a tensor for each relation of interest to return high scores when combined with the vectors of two entities that hold the intended relation. At test time, the system is used to classify relational tuples as true or false, as well as to predict new entities that hold a certain relationship with a target entity. This is quite close in spirit to what we do, except that, given an entity1-relation-entity 2 tuple, we treat relation-entity 2 as a binary attribute of entityl, and we try to induce such attributes on a larger scale (Socher et al. consider seven relations in total). Moreover, we rely on the same architecture to learn discrete features denoting relations with entities and numerical features, to induce full attribute-based descriptions of entities.

Our proposal is only distantly related to methods to embed words tokens and $\mathrm{KB}$ entities and relationships in a vector space, e.g., for better relation extraction (see Weston et al. (2013) and references therein). This line of work does not use distributional semantics to induce word vectors, and ignores numerical attributes.

The broader goal of getting at referential information with distributional semantics is shared with Herbelot (2015). However, the specific approach is different, as she constructs vectors for individual entities (literary characters) by contextualizing generic noun vectors with distributional properties of those entities. Finally, we share our methodology with work on mapping between corpus-based word representations and other representational spaces, such as subject-generated concept properties (Johns and Jones, 2012; Hill et al., 2014; Făgărăşan et al., 2015), visual features (Frome et al., 2013; Socher et al., 2013b; Lazaridou et al., 2014) or brain signals (Mitchell et al., 2008; Murphy et al., 2012). In all these settings, the focus is entirely on predicting numerical attributes, whereas we treat both numerical and binary attributes. Rubinstein et al. (2015) use distributional vectors to predict binary conceptual attributes of common nouns, as well as a continuous score measuring saliency of such attributes. Our target features are conceptually very different from those of all these studies.

\section{Discussion and Conclusion}

We have shown that a simple model can learn to predict, to a reasonable degree of accuracy, ref- 
erential attributes of an entity that are typically seen in a knowledge base from the corresponding corpus-based distributional representation. The results suggest that, while distributional semantic vectors can be used "as-is" to capture generic word similarity, with some supervision it is also possible to extract other kinds of information from them, including structured factual statements of the sort encoded in manually-curated knowledge bases. This makes distributional vectors very attractive as general-purpose word meaning representations.

We have also shown that some of the errors in the predictions can be explained on cultural grounds, but that these effects are more pronounced in the input of our model, a standard distributional semantic model, than in its output. In this sense, our model manages to objectify the information that it is provided with. Our analyses also suggest that the main limiting factor in learning referential attributes, apart from good old data sparseness, is the degree to which they are contextually supported, that is, to what extent they are expressed with consistent and specific linguistic means in the context of their target words. This determines whether they are actually represented in the distributional model in the first place.

More generally, we see our work as a small step towards the more general goal of bridging the concept-referent gap in distributional semantics. A common noun such as dog denotes a concept, based on a prototype with fuzzy boundaries, susceptible of metaphorical extensions, and bearing all the other hallmarks of generic conceptual knowledge (Carlson, 2009; Murphy, 2002). These might be adequately captured by the properties of the $d o g$ vector in distributional semantic space. However, when used in a specific discourse, words and more complex linguistic expressions often denote specific referents with fixed, "hard" properties, such as this dog, or Amur, when used for my neighbor's dog at 3.31pm on May 29th 2015 in Novosibirsk, a $61 \mathrm{~cm}$-tall black-and-tan foxhound. Amur is more easily characterized by a set of precise attributevalue pairs than by a vector in a generic conceptual space. Our experiment suggests that distributional vectors encode both generic conceptual knowledge and more precise attributes of specific referents. Of course, while we can use FreeBase and other knowledge bases to gather training data about public-domain entities, such as countries or cities, it is still not clear where we could gather appropriate training data to learn about the specific properties of "private-discourse" referents such as Amur. Moreover, it remains to be seen whether the properties of common named entities, such as countries and cities, that are in a sense "hybrid" between the conceptual and referential domains, also transfer to entities of a more specific and private kind. Finally, it is still not clear how to extend the current approach beyond words and phrases directly denoting an entity (Amur) to other kinds of definite descriptions (this dog).

Acknowledgments: This project has received funding from the European Union's Horizon 2020 research and innovation programme under the Marie Sklodowska-Curie grant agreement No 655577 (LOVe); ERC 2011 Starting Independent Research Grant n. 283554 (COMPOSES); DFG (SFB 732, Project D10); and Spanish MINECO (grant FFI2013-41301-P). This paper reflects the authors' view only, and the EU is not responsible for any use that may be made of the information it contains. Special thanks to Christian Scheible for help with the Machine Learning part, to the anonymous reviewers for insightful and constructive feedback, and to the FLOSS reading group for helping us shape our ideas on the topic of this paper.

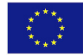

\section{References}

Marco Baroni and Alessandro Lenci. 2010. Distributional Memory: A general framework for corpus-based semantics. Computational Linguistics, 36(4):673-721.

Paul Buitelaar and Philipp Cimiano. 2008. Bridging the Gap between Text and Knowledge. IOS, Amsterdam.

Greg Carlson. 2009. Generics and concepts. In Jefrey Pelletier, editor, Kinds, Things and Stuff, pages 1635. Oxford University Press, Oxford, UK.

Bradley Efron and Robert Tibshirani. 1994. An Introduction to the Bootstrap. Chapman and Hall, Boca Raton, FL.

Katrin Erk, Sebastian Padó, and Ulrike Padó. 2010. A flexible, corpus-driven model of regular and inverse selectional preferences. Computational Linguistics, 36(4):723-763.

Katrin Erk. 2012. Vector space models of word meaning and phrase meaning: A survey. Language and Linguistics Compass, 6(10):635-653. 
Luana Făgărăşan, Eva Maria Vecchi, and Stephen Clark. 2015. From distributional semantics to feature norms: grounding semantic models in human perceptual data. In Proceedings of IWCS, pages 52 57, London, UK.

André Freitas and Edward Curry. 2014. Natural language queries over heterogeneous linked data graphs: A distributional-compositional semantics approach. In Proceedings of IUI, pages 279-288, Haifa, Israel.

André Freitas, João Carlos Pereira da Silva, Edward Curry, and Paul Buitelaar. 2014. A distributional semantics approach for selective reasoning on commonsense graph knowledge bases. In Proceedings of $N L D B$, pages 21-32, Montpellier, France.

Alinda Friedman, Dennis Kerkman, and Norman Brown. 2002. Spatial location judgments: A crossnational comparison of estimation bias in subjective North American geography. Psychonomic Bulletin \& Review, 9(3):615-623.

Andrea Frome, Greg Corrado, Jon Shlens, Samy Bengio, Jeff Dean, Marc'Aurelio Ranzato, and Tomas Mikolov. 2013. DeViSE: A deep visual-semantic embedding model. In Proceedings of NIPS, pages 2121-2129, Lake Tahoe, NV.

Aurélie Herbelot. 2015. Mr Darcy and Mr Toad, gentlemen: distributional names and their kinds. In Proceedings of IWCS, pages 151-161, London, UK.

Felix Hill, Roi Reichart, and Anna Korhonen. 2014. Multi-modal models for concrete and abstract concept meaning. Transactions of the Association for Computational Linguistics, 2:285-296.

Brendan Johns and Michael Jones. 2012. Perceptual inference through global lexical similarity. Topics in Cognitive Science, 4(1):103-120.

Willis F. Kern and James R. Bland. 1948. Solid Mensuration with Proofs. Wiley, New York, 2nd edition.

Angeliki Lazaridou, Elia Bruni, and Marco Baroni. 2014. Is this a wampimuk? Cross-modal mapping between distributional semantics and the visual world. In Proceedings of ACL, pages 1403-1414, Baltimore, MD.

Max Louwerse and Rolf Zwaan. 2009. Language encodes geographical information. Cognitive Science, 33:51-73.

Chris Manning, Prabhakar Raghavan, and Hinrich Schütze. 2008. Introduction to Information Retrieval. Cambridge University Press, Cambridge, UK.

Tomas Mikolov, Wen-tau Yih, and Geoffrey Zweig. 2013. Linguistic regularities in continuous space word representations. In Proceedings of NAACL, pages 746-751, Atlanta, Georgia.
Mike Mintz, Steven Bills, Rion Snow, and Dan Jurafsky. 2009. Distant supervision for relation extraction without labeled data. In Proceedings of $A C L$, pages 1003-1011, Singapore.

Tom Mitchell, Svetlana Shinkareva, Andrew Carlson, Kai-Min Chang, Vincente Malave, Robert Mason, and Marcel Just. 2008. Predicting human brain activity associated with the meanings of nouns. Science, 320:1191-1195.

Brian Murphy, Partha Talukdar, and Tom Mitchell. 2012. Selecting corpus-semantic models for neurolinguistic decoding. In Proceedings of *SEM, pages 114-123, Montreal, Canada.

Gregory Murphy. 2002. The Big Book of Concepts. MIT Press, Cambridge, MA.

Sebastian Padó and Mirella Lapata. 2007. Dependency-based construction of semantic space models. Computational Linguistics, 33(2):161-199.

Mark Palatucci, Dean Pomerleau, Geoffrey Hinton, and Tom Mitchell. 2009. Zero-shot learning with semantic output codes. In Proceedings of NIPS, pages 1410-1418, Vancouver, Canada.

Dana Rubinstein, Effi Levi, Roy Schwartz, and Ari Rappoport. 2015. How well do distributional models capture different types of semantic knowledge? In Proceedings of ACL (Volume 2: Short Papers), pages 726-730, Beijing, China.

Richard Socher, Danqi Chen, Christopher Manning, and Andrew Ng. 2013a. Reasoning with neural tensor networks for knowledge base completion. In Proceedings of NIPS, pages 926-934, Lake Tahoe, NV.

Richard Socher, Milind Ganjoo, Christopher Manning, and Andrew Ng. 2013b. Zero-shot learning through cross-modal transfer. In Proceedings of NIPS, pages 935-943, Lake Tahoe, NV.

Peter Turney and Patrick Pantel. 2010. From frequency to meaning: Vector space models of semantics. Journal of Artificial Intelligence Research, 37:141-188.

Jason Weston, Antoine Bordes, Oksana Yakhnenko, and Nicolas Usunier. 2013. Connecting language and knowledge bases with embedding models for relation extraction. In Proceedings of EMNLP, pages 1366-1371, Seattle, WA. 